\title{
ELECCIONES REGIONALES FRANCESAS DE 22 DE MARZO DE 1992
}

\author{
CARMEN FERNÁNDEZ-MIRANDA CAMPOAMOR \\ Profesora Titular de Derecho Constitucional \\ Universidad Nacional de Educación a Distancia
}


SUMARIO

I. Introducción.-II. Campaña electoral.-III. Resultados electoraLES.-IV. Valoración. 
Revista de Derecho Político, núm. 38, 1994, pp. 377-392

\title{
ELECCIONES REGIONALES FRANCESAS DE 22 DE MARZO DE 1992
}

\author{
POR \\ CARMEN FERNÁNDEZ-MIRANDA CAMPOAMOR \\ Profesora Titular de Derecho Constitucional de la UNED
}

\section{INTRODUCCIÓN}

El domingo 22 de marzo de 1992 se celebran en Francia las segundas elecciones regionales ', a fin de renovar, por sufragio universal directo, a una vuelta y con un sistema proporcional de escrutinio, los 26 Consejos Regionales ${ }^{2}$ del territorio metropolitano y de ultramar. El viernes siguiente -26 de marzo- se constituyen los órganos y los Consejeros eligen a su Presidente.

1 Las primeras se celebraron el 16 de marzo de 1986. El día 22 de marzo se realiza también la primera vuelta para la elección de los Consejos Generales; la segunda vuelta es el 29 de marzo; en estas elecciones cantonales se utiliza el sistema de escrutinio mayoritario.

2 Los Consejos Regionales son establecidos por la Ley de descentralización de marzo de 1982, cuyo propulsor, el Ministro del Interior, Gaston Defferre, pretendía "situar a Francia en la hora de la Europa de las Regiones"; sin embargo, esta norma ha sido muy criticada por quedarse a medio camino entre el centralismo clásico francés y las autonomías políticas de otros países europeos, creando unos organismos con escasas y difusas competencias; entre éstas: los centros de formación profesional e institutos de bachillerato, carreteras, asistencia social, protección del medio ambiente y promoción del desarrollo económico. "La derrota de las Regiones", El País, 22 de marzo de 1992. "El vecino François Mitterrand", Diario 16, 20 de noviembre de 1991. R. Cotarelo: "La caída del socialismo galo», Diario 16, 12 de enero de 1992. 
La trascendencia política de esta elección no radica en la importancia de los organismos regionales, sino en la variación en el reparto de poder, anunciada por todos los sondeos electorales, de cara a las próximas elecciones legislativas de 1993; desde todos los sectores se valora esta consulta electoral como un indicador de la voluntad del electorado que retira su apoyo al partido del Gobierno y del Presidente de la República.

El ambiente social es tenso, con un paro creciente, una población inmigrante cercana al $4 \%$ y continuas acusaciones de corrupción política y financiera que afectan esencialmente al Partido Socialista, que ocupa en los sondeos las cotas más bajas de popularidad. Todos estos factores crean una gran preocupación ciudadana ante la inminente integración en Europa, con una Alemania unida, la aparición de múltiples Estados consecuencia del desastre comunista y, en casa, el imparable descenso del partido en el poder desde $1981^{3}$.

\section{Cuadro 1}

Principales partidos que compiten en estas elecciones y sus lideres

\begin{tabular}{|c|c|c|}
\hline Siglas & Partídos politicos & Lideres \\
\hline $\begin{array}{l}\text { PSF } \\
\text { FN }\end{array}$ & $\begin{array}{l}\text { Partido Socialista Francés } \\
\text { Frente Nacional }\end{array}$ & $\begin{array}{l}\text { F. Mitterrand } \\
\text { J. M. Le Pen }\end{array}$ \\
\hline $\begin{array}{l}\text { UPF } \\
\text { UDF }\end{array}$ & $\begin{array}{l}\text { Unión Para Francia, coalición que agrupa a: } \\
\text { Unión para la Democracia Francesa }\end{array}$ & V. Giscard d'Estaing \\
\hline RPR & 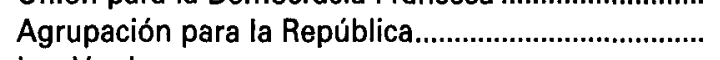 & J. Chirac \\
\hline LV & 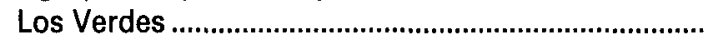 & R. Waechter \\
\hline GE & 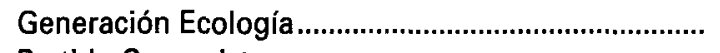 & B. Lalonde \\
\hline PCF & Partido Comunista & G. Marchais \\
\hline
\end{tabular}

3 Los medios de comunicación critican la inusitada falta de reflejos del Presidente Mitterrand ante los últimos acontecimientos europeos tanto respecto a la unificación alemana como a la desaparición de la URSS y el protagonismo creciente de Yeltsin; la última crisis (febrero de 1992), producida por el "caso Habache", fue considerada como el ridículo francés en el exterior. Ver "El último coletazo de Mitterrand", en El País, editorial, 12 de septiembre de 1991. R. CotARelo: "Francia: hacia la catástrofe», Diario 16, 23 de febrero de 1992. 


\section{CAMPAÑA ELECTORAL}

Los sondeos de opinión publicados por la prensa ${ }^{4}$ manifiestan una modificación sustancial del mapa político francés, con una intención de voto que se puede resumir de la siguiente forma: descenso de los partidos protagonistas de la V República, importantísimo para el PS 117 a $21 \%$ ) y moderado para la derecha RPR-UDF (33 a $34 \%$ ); a su vez, ascenso relevante del Frente Nacional -hasta el 15\% - y de los Grupos Ecologistas, un $8 \%$ para los Verdes y entre el 6 y $7 \%$ para Generación Ecología ${ }^{5}$. No hay que olvidar la enorme abstención que se anuncia, del $54 \%$ al inicio de la campaña y entre el $47 \%$ y el $52 \%$ la víspera de la votación.

Estos datos sobre las expectativas de voto suponen un gran impacto sobre la clase política y el electorado, magnificando de tal modo la consulta electoral del 22 de marzo, que se interpretan todos los indicios como el fin de la era Mitterrand ${ }^{6}$.

El Partido Socialista (PSF) afronta la campaña electoral desde un triple fracaso de su programa: social, no sólo no ha corregido las desigualdades sociales, sino que el paro aumenta de forma alarmante; moral, continuos escándalos políticos y financieros, e ideológico, transformaciones cada vez peor aceptadas por su electorado, escéptico ante un partido teóricamente de izquierdas pero cada vez más alejado del ideal de cambio social que les dio el triunfo en $1981^{\prime}$. Consciente del abandono progresivo de sus votantes ${ }^{8}$, intenta recuperarlos centrando la campaña en torno a la unión frente al polémico partido de Le Pen?

4 Ver, para datos puntuales y en distintas fechas: Libération, 13 de febrero de 1992; Libération, 2 de marzo de 1992; Paris-Match, 9-15 de marzo de 1992; Le Point, 16-22 de marzo de 1992.

5 El electorado manifiesta su simpatía por los Grupos Ecologistas (71\%); sin embargo, no como partidos gobernantes, sino como grupos de presión. Sondeos cit.

J. M. Colombanı, en Le Monde, manifiesta cómo Mitterrand ha pasado de ser un elemento de estabilidad política a inquietar profundamente al electorado: $\mathrm{J}$. VALENZUela: "Mitterrand desciende a los infiernos", en El País, 1 de marzo de 1992. Igualmente J. DANIEL, director de Le Nouvel Observateur, escribe que "a los franceses les gustaría ya cambiar de jefe", en "Las eternas fantasías de los galos", El País, 11 de marzo de 1992.

7 El elector socialista ha visto el largo camino recorrido por este partido, desde la alianza con el PC y las numerosas nacionalizaciones del primer período de gobierno hasta la progresiva aceptación del libre mercado; en las declaraciones de E. Cresson a la prensa con motivo del inicio de su mandato, manifiesta que la socialdemocracia es una buena fórmula.

${ }_{8}$ El Presidente Mitterrand ha realizado, desde hace un año, numerosos movimientos tendentes a la cohesión de su partido de cara a las actuales eleccio- 
El partido neogaullista de J. Chirac (RPR) y el centrista de V. Giscard d'Estaing (UDF) forman una coalición -UPF- para presentarse unidos en todas las listas. Pese a que los sondeos les colocan en primer lugar en las preferencias del electorado, mantienen una actitud prudente, pues pierden votos en relación a otras convocatorias, con lo que no parece que sea su programa el que vaya a atraer al elector socialista desengañado. Durante toda la campaña se desmarcan del Frente Nacional, manifestando su intención de no pactar con este partido aun a riesgo de perder algún Consejo Regional.

El Partido Comunista (PC), pese a la crisis ideológica por la que atraviesa, se mantiene estable en los sondeos; todos sus esfuerzos se dirigen a captar al elector socialista más radical.

El Frente Nacional (FN) concita las críticas de todo el espectro político por su virulencia; Le Pen aprovecha la difícil situación por la que atraviesa el país para lanzar el mensaje de "ley, orden y pureza patriótica" ${ }^{10}$, claramente racista y xenófobo. El importante número de votos que le deparan los sondeos le sitúan en una posición triunfalista y agresiva, con todo tipo de incidentes violentos en los mítines que protagoniza su líder.

Finalmente, el ecologismo se presenta con dos alternativas rivales, que parecen ser el refugio de los desencantados: el ecologismo radical de "los verdes", de A. Waetcher y Generación Ecología, del Ministro de Medio Ambiente, B. Lalonde, que pretende una uecología política alejada de las utopías".

nes $y$, esencialmente, a las próximas legislativas; en esta dinámica puede señalarse el relevo de $P$. Mauroy al frente de la Secretaría General, siendo sustituido por $L$. Fabius (7-IV-1991), buscando una nueva imagen del partido; igualmente, el leve giro a la izquierda que supone el cambio de M. Rocard por E. Cresson (15-V-91). Junto a estos cambios personales, Mitterrand intenta atraer la atención del ciudadano con el anuncio de medidas espectaculares (10-XI-1991), tales como la reforma de la Constitución - reducción del mandato presidencial, potenciación de la Asamblea Nacional y más competencias para el Consejo Constitucional- y la modificación del actual sistema de escrutinio mayoritario de las elecciones legislativas, por un sistema mixto con fuertes dosis de proporcionalidad que impediría a la derecha lograr una mayoría suficiente en 1993. Al Presidente Mitterrand no se le ha olvidado la mayor humillación de su largo mandato: la cohabitación 1986-1988, y creo que utilizará todos los medios para impedirla, salvo uno: su dimisión.

9 P. GonZÁlEZ: "El extraño maridaje francés de socialistas y neofascistas", en Diario 16, 4 febrero 1992.

10 Editorial Diario 16, 22 marzo 1992. 
La víspera electoral los líderes políticos llaman a los ciudadanos a las urnas, para evitar la gran abstención que se anuncia, que favorecería fundamentalmente al Frente Nacional. La ascensión de este partido es el punto de atención de Francia y de Europa.

\section{RESULTADOS ELECTORALES}

\section{Cuadro 2}

Datos totales del Ministerio del Interior "1

\begin{tabular}{|c|c|c|}
\hline & Totales & $\%$ \\
\hline Inscritos & 37.344 .864 & \\
\hline 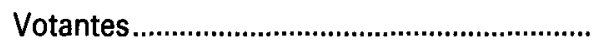 & 25.652 .385 & 68,7 \\
\hline Expr & 24.431 .676 & 65,4 \\
\hline Abst. & & 31,3 \\
\hline 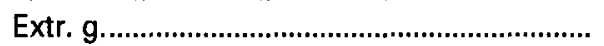 & 298.643 & 1,2 \\
\hline PC & 1.963 .562 & 8,0 \\
\hline PS & 4.468 .849 & 18,3 \\
\hline 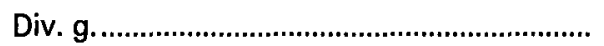 & 523.070 & 2,1 \\
\hline GE & 1.744 .350 & 7,1 \\
\hline Verdes & 1.659 .798 & 6,8 \\
\hline 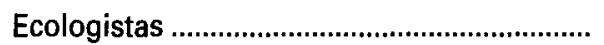 & 184.916 & 0,8 \\
\hline 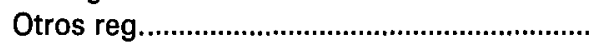 & 108.549 & 0,4 \\
\hline Categ. & 40.017 & 0,9 \\
\hline 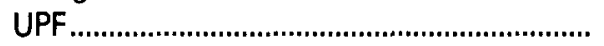 & 8.071 .623 & 33,0 \\
\hline 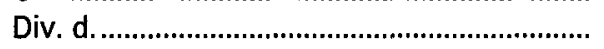 & 1.021 .079 & 4,2 \\
\hline FN & 3.396 .141 & 13,9 \\
\hline 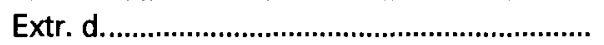 & 48.879 & 0,2 \\
\hline
\end{tabular}

\section{VALORACIÓN}

Estas elecciones han supuesto, efectivamente, "el estallido del paisaje político" ${ }^{12}$. Los sondeos han permitido al electorado conocer el panorama general pronosticado $y$, en consecuencia, ajustar su voto: acu-

1 Datos oficiales facilitados por la Embajada francesa.

12 S. JuLl: "El fin de la era Mitterrand", El Mundo, 24 marzo 1992. Ver datos puntuales en Le Monde, 24 marzo 1992. 
diendo masivamente a las urnas y estabilizando el peligroso ascenso del Frente Nacional.

El Partido Socialista cosecha el desastre que se venía anunciando con un $18,3 \%$ de votos; sin embargo, su fracaso no ha beneficiado a la coalición UPF, que, pese a ser la minoría mayoritaria - con un 33\%-, también sufre un desgaste. Con ello, el electorado ha castigado a los partidos clásicos: un Partido Socialista dividido y corrompido, en lucha por la sucesión de Mitterrand; una derecha que, pese a ir unida en esta ocasión, se muestra divergente en numerosas cuestiones puntuales. Todos los comentaristas políticos ven en estos resultados la existencia de una crisis politica ${ }^{13}$.

F. REVEL, en una sugestiva interpretación de los resultados, entiende que éstos son un voto de mensaje y no un voto de gobierno, una advertencia del electorado a los grandes partidos ${ }^{14}$.

Pese a todo, la coalición UPF se muestra eufórica con ese $33 \%$ de respaldo ciudadano, convencida de la eficacia de la unión de cara al triunfo del 93; reitera su promesa de no pactar con Le Pen en las negociaciones post-electorales.

El Frente Nacional gana votos ${ }^{15}$, pero no en la proporción que esperaba, a lo que contribuye la gran participación del electorado $-68,7 \%$ consciente de que en esta votación se dirimía algo más que unas elecciones regionales; pero con ese $13,9 \%$ se convierte en el primer partido europeo de esta ideología y ocupa la segunda posición en regiones de la importancia de Provenza-Alpes-Costa Azul, Ródano-Alpes e Isla de Francia.

Los resultados electorales suponen la entrada en política del ecologismo, pasando de un porcentaje mínimo a cerca del $14 \%$ en conjunto: Generación Ecología el $7,1 \%$ y Los Verdes el $6,8 \%$. Su éxito se debe a haber atraído al electorado procedente del socialismo, escéptico ante su olvidada promesa de cambiar la sociedad, oferta que parece corresponden a los movimientos ecológicos. El porcentaje de votos alcanzado coloca a estos partidos en bisagra de la política francesa $y$, si son capaces de superar sus rivalidades y prescindir de proyectos irrealizables, en una im-

13 A. Touraine: "El sentido de un fracaso", El País, 25 marzo 1992. R. CotaRELo: "¿Quién ganó en Francia?", Diario 16, 29 marzo 1992. F. SAHAGÚN: "Ante un nuevo mapa electoral», El Mundo, 23 marzo 1992.

14 "Las elecciones de las regionales francesas", El Mundo, 24 marzo 1992.

15 Recoge el voto del miedo y del escepticismo ante el sistema democrático, consecuencia de la corrupción política y de la crisis ideológica que vive Occidente. Editorial de El Mundo, 23 marzo 1992. 
portante fuerza de cara al 93 . También los votos más radicales procedentes del socialismo, junto a los fieles comunistas incombustibles, han permitido a este partido estabilizarse, aunque claramente a la baja ( $8 \%)$, y no habiendo logrado superar el límite del $10 \%$ que se había propuesto.

\section{Cuadro 3}

Evolución del voto ${ }^{16}$

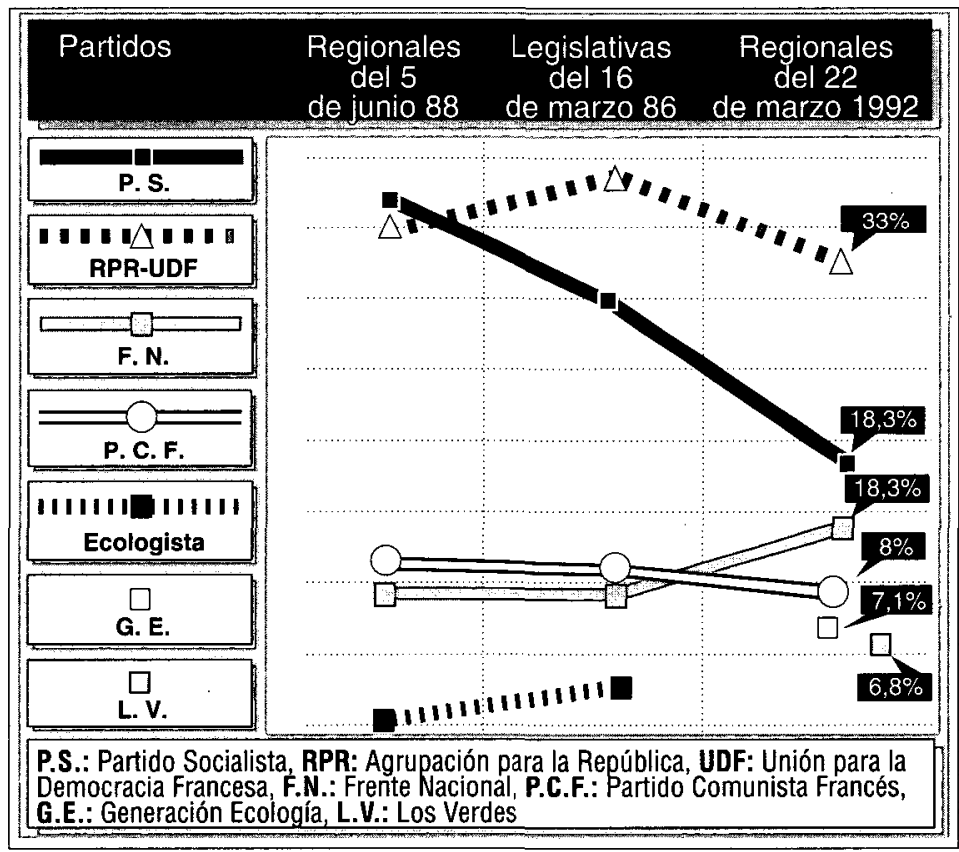

Los resultados de la votación del día 22 sólo arrojan dos mayorías absolutas de la coalición UPF: Auvernia y Loira; en el resto de los Consejos son necesarios los pactos para la elección de Presidentes, que se realizará por mayoría relativa en tercera votación, con el riesgo de ingobernabilidad correspondiente. 
En estas negociaciones hay que señalar dos fracasos: el partido socialista no logra ser apoyado por los ecologistas, como esperaba " coalición UPF mantiene su promesa electoral de no pactar con el partido de Le Pen, a diferencia de lo hecho en las regionales de $1986^{18}$.

El Partido Socialista consigue, en principio, la presidencia de Limousin y las conflictivas de Borgoña y Lorena. En Borgoña es elegido Presidente J. P. Soisson, Ministro de la Función Pública; en Lorena, J. M. Rausch, Ministro de Comunicaciones, ambos candidatos del PS y ambos acusados de haber logrado la elección gracias a los votos del Frente $\mathrm{Na}$ cional. Ante el escándalo generado y recogido en toda la prensa ${ }^{19}$, la Primera Ministra, E. Cresson, les exige el abandono de tal presidencia, lo que hará Rausch pero no Soisson, que dimite como Ministro. La coalición UPF, a su vez, tras las negociaciones, logra presidir los siguientes Consejos: Baja Normandia, Bretaña, Picardía, Isla de Francia, Champaña-Ardenas, Alsacia, Centro, Poitou-Charente, Aquitania, Midi-Pirineos, Languedoc-RoseIlón, Provenza-Alpes-Costa Azul y Ródano-Alpes ${ }^{20}$. Es aplazada la decisión hasta el día 30 de marzo en Alta Normandía (vencerá UPF), Franco Condado (también vencerá UPF) y Norte-Paso de Calais, principal feudo socialista, en donde este partido, ante la imposibilidad de conseguir apoyos, votará sorpresivamente a la candidata de Los Verdes ${ }^{21}$. En resumen, dos Consejos presididos por el PS, uno por LV y el resto por UPF.

El caso de Córcega es especial, pues participa en las elecciones de forma distinta al resto, debido a su estatuto especial. La elección se celebra a dos vueltas (22 y 29 de marzo) y con un sistema de escrutinio mayoritario. Hay que señalar el importante ascenso de los nacionalistas $E$. Simeoni (Nación Córcega) y O. Orsini (Movimiento por la Autodeterminación), que obtienen un porcentaje del $25 \%$ en conjunto; sin embargo, los partidos estatales partidarios de una Córcega francesa logran el $34 \%$ de los votos ${ }^{22}$.

17 Ni siquiera por Generación Ecología, cuyo líder, Brice Lalonde, es Ministro de Medio Ambiente en el Gobierno de E. Cresson, puesto del que dimite voluntariamente para dedicarse a su partido.

Hay que recordar también que en Francia no existe incompatibilidad entre los cargos políticos estatales y locales; esto es otro dato para explicar la relevancia que siempre tienen este tipo de elecciones, al margen de esta ocasión concreta.

${ }_{18}$ Esto le resulta eficaz en un doble sentido: de cara a su electorado y de cara a sus propios intereses, ya que no sufre ninguna pérdida al no ser apoyado el PS por los Ecologistas.

19 Ver prensa francesa y española del día 28 de marzo de 1992, y El Mundo y El País del 29 de marzo.

20 El País, 28 marzo 1992.

21 El País, 31 marzo 1992.

22 El País, 31 marzo 1992. 


\section{Cuadro 4}

Las elecciones por regiones ${ }^{23}$

\begin{tabular}{l} 
Partido \\
\hline PARIS \\
París como provincia electoral no existe, forma parte \\
de la región de l'Île de France". Pero los resultados \\
son muy significativos. Le Pen consiguió el 2. puesto.
\end{tabular}

\section{BAJA NORMANDIA}

PS

PC.

UPF

ECOL.

FN

7,20

15,30

F

\section{ALTA NORMANDÍA}

PS.

PC

41,10

ECOL

3,50

7,10

FN

\section{PICARDIA}

PS.

PC.

\section{ECOL}

9,60

14,90

FN

\section{LOIRA}

PS.

PC.

ECOL

\section{NORTE-PASO DE CALAIS}

PS.

PC.

UPF

ECOL 
Cuadro 4 (Cont.)

Las elecciones por regiones

\begin{tabular}{|c|c|c|}
\hline Partido & 86 & 92 \\
\hline \multicolumn{3}{|l|}{ CHAMPAGNE-ARDENAS } \\
\hline 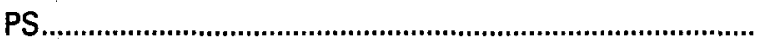 & 29,68 & 16,79 \\
\hline 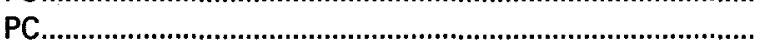 & 11,07 & 7,75 \\
\hline UPF & 35,41 & 27,62 \\
\hline ECOL. & 1,01 & 16,20 \\
\hline FN & 9,60 & 18,41 \\
\hline \multicolumn{3}{|l|}{ LORENA } \\
\hline PS & 28,90 & 14,40 \\
\hline PC & 7,80 & 5,90 \\
\hline 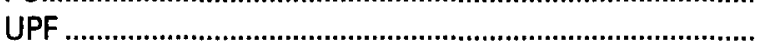 & 37,10 & 31,30 \\
\hline ECOL. & 2,50 & 17,00 \\
\hline FN & 10,40 & 14,60 \\
\hline \multicolumn{3}{|l|}{ BRETAÑA } \\
\hline PS.................. & 32,60 & 19,59 \\
\hline 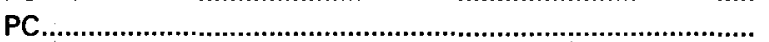 & 7,03 & 4,94 \\
\hline UPF & 22,92 & 36,91 \\
\hline ECOL & 2,36 & 15,80 \\
\hline FN & 5,04 & 9,08 \\
\hline \multicolumn{3}{|l|}{ ALSACIA } \\
\hline 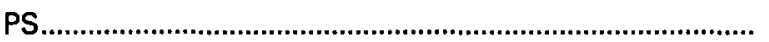 & 19,49 & 12,31 \\
\hline PC & 2,03 & 1,63 \\
\hline UPF & 46,86 & 33,08 \\
\hline 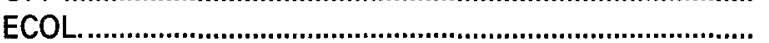 & 6,06 & 16,81 \\
\hline FN & 13,02 & 17,16 \\
\hline \multicolumn{3}{|l|}{ POITOU-CHARENTES } \\
\hline PS. & 34,00 & 21,30 \\
\hline 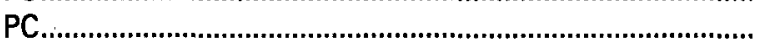 & 9,00 & 7,00 \\
\hline 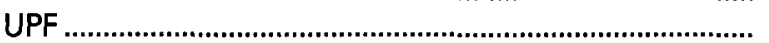 & 41,60 & 37,50 \\
\hline ECOL & - & 18,40 \\
\hline FN & 5,60 & 8,90 \\
\hline \multicolumn{3}{|l|}{ AQUITANIA } \\
\hline$\ldots$ & 34,78 & 20,75 \\
\hline 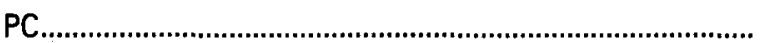 & 9,20 & 7,65 \\
\hline UPF & 41,41 & 31,07 \\
\hline ECOL. & 4,68 & 10,00 \\
\hline FN & 7,01 & 10,00 \\
\hline
\end{tabular}


Cuadro 4 (Cont.)

Las elecciones por regiones

\begin{tabular}{|c|c|c|}
\hline Partido & 86 & 92 \\
\hline \multicolumn{3}{|l|}{ AUVERNIA } \\
\hline 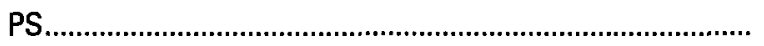 & 30,74 & 18,96 \\
\hline PC & 11,19 & 9,99 \\
\hline UPF & 46,52 & 42,64 \\
\hline ECOL. & 1,78 & 11,65 \\
\hline FN & 5,93 & 8,97 \\
\hline \multicolumn{3}{|l|}{ PROVENZA-ALPES-C. AZUL } \\
\hline 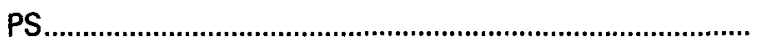 & 24,00 & 20,07 \\
\hline PC & 12,00 & 8,06 \\
\hline UPF & 36,07 & 29,02 \\
\hline ECOL & 2,05 & 9,04 \\
\hline FN & 20,00 & 23,04 \\
\hline \multicolumn{3}{|l|}{ FRANCO-CONDADO } \\
\hline 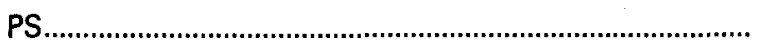 & 33,90 & 16,70 \\
\hline PC & 6,80 & 4,00 \\
\hline UPF & 40,60 & 35,70 \\
\hline ECOL. & 3,00 & 15,80 \\
\hline FN & 9,50 & 12,50 \\
\hline \multicolumn{3}{|l|}{ LIMOUSIN } \\
\hline 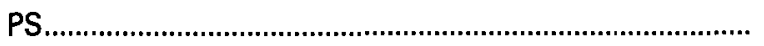 & 31,00 & 25,80 \\
\hline PC & 19,50 & 10,20 \\
\hline UPF & 40,00 & 36,40 \\
\hline ECOL. & 1,40 & 10,00 \\
\hline FN & 3,90 & 6,50 \\
\hline \multicolumn{3}{|l|}{ LANGUEDOC-ROSELLON } \\
\hline PS & 31,00 & 19,40 \\
\hline PC & 14,70 & 11,30 \\
\hline UPF & 34,60 & 27,70 \\
\hline ECOL. & 1,20 & 12,80 \\
\hline FN & 13,00 & 17,40 \\
\hline \multicolumn{3}{|l|}{ ILE DE FRANCE } \\
\hline 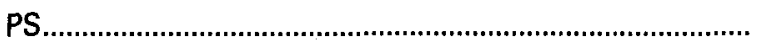 & 59 & 32 \\
\hline PC & 19 & 16 \\
\hline UPF & 84 & 80 \\
\hline ECOL & 一 & 37 \\
\hline FN & 19 & 37 \\
\hline
\end{tabular}


Cuadro 4 (Cont.)

Las elecciones por regiones

\begin{tabular}{|c|c|c|}
\hline Partido & 86 & 92 \\
\hline \multicolumn{3}{|l|}{ CENTRO } \\
\hline PS & 20 & 17 \\
\hline PC & 8 & 8 \\
\hline UPF & 28 & 27 \\
\hline ECOL & - & 8 \\
\hline FN & 3 & 11 \\
\hline \multicolumn{3}{|l|}{ BORGOÑA } \\
\hline PS & 18 & 8 \\
\hline PC & 5 & 3 \\
\hline UPF & 24 & 33 \\
\hline ECOL. & - & 7 \\
\hline FN & 3 & 8 \\
\hline \multicolumn{3}{|l|}{ RODANO-ALPES } \\
\hline PS & 43 & 23 \\
\hline PC & 13 & 11 \\
\hline UPF & 67 & 62 \\
\hline ECOL & - & 21 \\
\hline FN & 13 & 29 \\
\hline \multicolumn{3}{|l|}{ PIRINEOS CENTRALES } \\
\hline PS & 27 & 27 \\
\hline PC & 5 & 5 \\
\hline UPF & 32 & 38 \\
\hline ECOL & - & 7 \\
\hline FN & 1 & 6 \\
\hline
\end{tabular}




\section{Cuadro 5}

Presidencias de las regiones francesas ${ }^{24}$

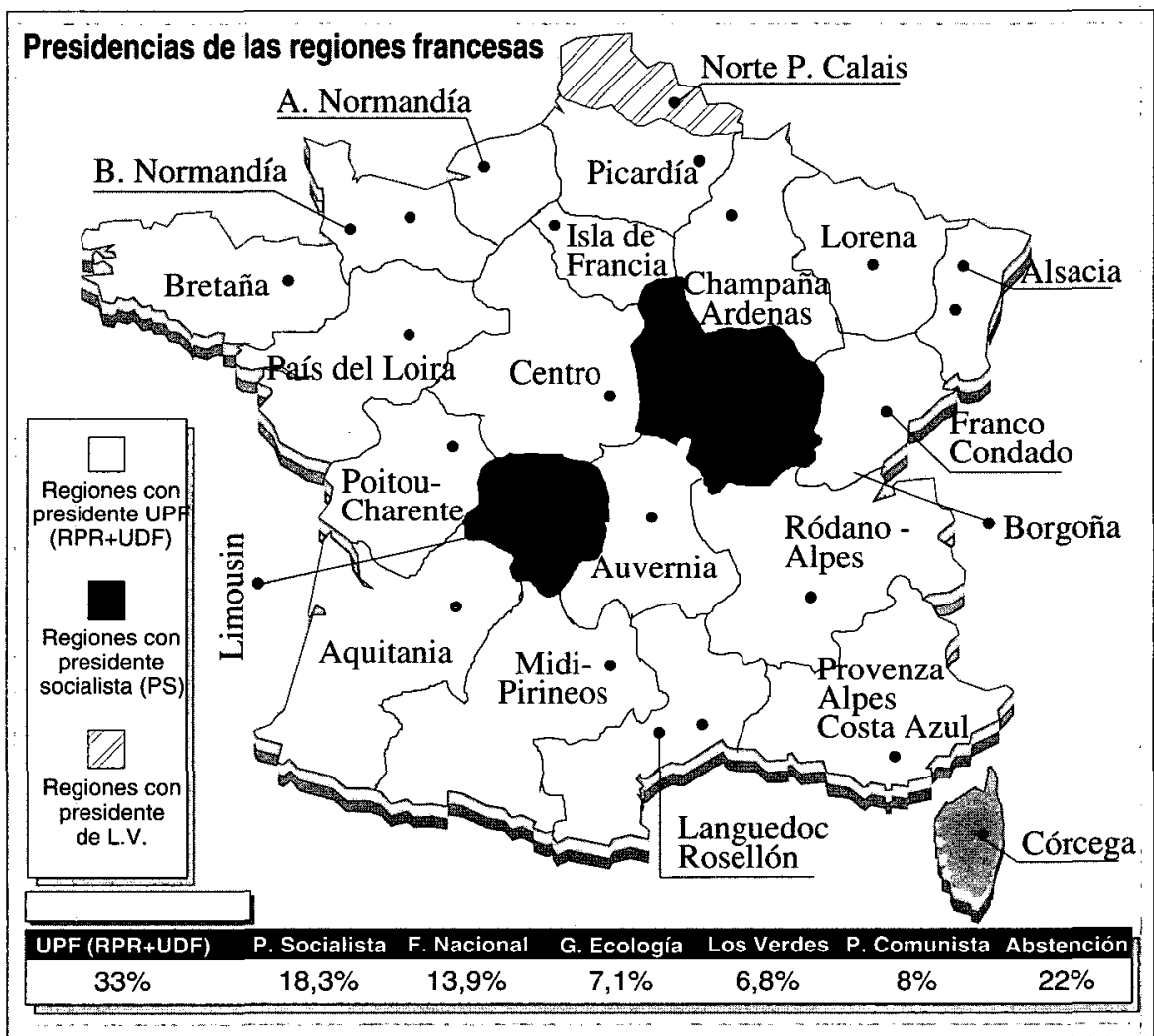

Como conclusión, el electorado francés se ha alejado esencialmente del partido en el poder. Tras estos resultados se da la paradoja de que un partido con un respaldo del $18,3 \%$ ocupa las instituciones del Estado y ha de acometer la integración europea ${ }^{25}$. Pese a la presión de la opinión pública y la prensa de todo el país responsabilizando al Presidente de la situación política en que se encuentra Francia ${ }^{26}$, la reacción in-

24 Elaborado con datos publicados por El País, 28 marzo 1992.

25 Editorial de El País, 10 abril 1992.

${ }^{26}$ Ver Libération, Le Figaro, L'Humanité y Le Quotidien de Paris, 30 marzo 1992. Los sondeos publicados en estos días por la prensa francesa manifiestan que un $50 \%$ del electorado pide la disolución de la Asamblea Nacional, lo que coincide con la petición de UDF y RPR. 
mediata del Presidente Mitterrand no va a ser convocar elecciones legislativas - como pide interesadamente la coalición UPF- ni mucho menos reducir el mandato presidencial y autoaplicarse la reforma, sino, obsesionado por no dimitir y no soportar una nueva cohabitación (que se avecina si la coalición UPF triunfa en 1993), retira su confianza a la impopular Edith Cresson ${ }^{27}$ y mediante el nombramiento del Ministro de Finanzas, Beregevoy, como Primer Ministro, intenta recuperar la confianza del electorado socialista ${ }^{28}$. Otra posibilidad para impedir la mayoría de UPF en 1993 sería la reforma del sistema electoral, en contra de la opinión de los líderes más importantes del partido -Rocard, Jospin y Fabius ${ }^{29}$ - y el riesgo de que el Frente Nacional entre en la Asamblea con un importante número de diputados ${ }^{30}$. El Jefe del Estado se encuentra en una difícil situación política y habrá de valorar adecuadamente las decisiones que tome teniendo en cuenta el interés del país.

Sin embargo, y pese al panorama descrito sobre los días siguientes a las elecciones regionales, hay que tener en cuenta la integración en la Comunidad Europea y el debate nacional en torno a Maastricht. Esto podría ser una importante plataforma para el hábil político que es $\mathrm{F}$. Mitterrand y esto podría ser, al mismo tiempo, origen de múltiples problemas para la coalición de Chirac y Giscard, debido a los diferentes planteamientos de sus partidos en torno a múltiples cuestiones concretas. Las legislativas de 1993 están muy cerca o muy lejos, dependiendo de la óptica desde la que se mire.

Madrid, mayo de 1992

$27 \quad$ Dimite el 2 de abril de 1992.

28 Con ello pretende "movilizar a sus partidarios más próximos para un importantísimo asalto que debe evitale anticipar de un modo u otro el final de su mandato... Nombra un equipo homogéneo que reinvente un discurso de izquierda y vuelva a conferir identidad electoral a los desencantados del socialismo". S. JuLl, "El social-monetarismo", El Mundo, 5 abril 1992.

${ }^{29}$ No les interesa un sistema electoral que dificulte la obtención de mayorías de cara a las elecciones presidenciales que aún pretenden que gane su partido, para lo que buscan el pacto y la cohesión interna. El Sol, 10 enero 1992.

30 En El Mundo del 24 marzo de 1992 se hace una extrapolación de los actuales resultados a las legislativas próximas; con el actual sistema mayoritario UPF lograría 420 escaños y sólo 120 el PS. El sistema proporcional afectaria duramente a la derecha, pero el FN lograría nada menos que 77 escaños. 